\title{
Building and Testing a Statistical Shape Model of the Human Ear Canal
}

\author{
Rasmus Paulsen ${ }^{1}$, Rasmus Larsen ${ }^{1}$, Claus Nielsen ${ }^{2}$, \\ Søren Laugesen ${ }^{2}$, and Bjarne Ersbøll ${ }^{1}$ \\ 1 Informatics and Mathematical Modelling, Technical University of Denmark \\ Richard Petersens Plads, Building 321, DK-2800 Kgs. Lyngby, Denmark \\ \{rrp,rl, be\}@imm.dtu.dk, http://www.imm.dtu.dk/ \\ 2 Oticon Research Centre Eriksholm, Kongevejen 243, DK-3070 Snekkersten, \\ Denmark \\ $\{\mathrm{cni}, \mathrm{slu}\} @$ oticon.dk, http://www.oticon.com/
}

\begin{abstract}
Today the design of custom in-the-ear hearing aids is based on personal experience and skills and not on a systematic description of the variation of the shape of the ear canal. In this paper it is described how a dense surface point distribution model of the human ear canal is built based on a training set of laser scanned ear impressions and a sparse set of anatomical landmarks placed by an expert. The landmarks are used to warp a template mesh onto all shapes in the training set. Using the vertices from the warped meshes, a 3D point distribution model is made. The model is used for testing for gender related differences in size and shape of the ear canal.
\end{abstract}

\section{Introduction}

Hearing aids come in a number of different styles. The smallest of these styles is called CIC (Completely In the Canal). A CIC hearing aid consists of an acrylic shell containing microphone, amplifier, loudspeaker, and battery. It is placed completely in the ear canal rendering it invisible to an observer viewing the bearer from the front. This is cosmetically appealing, and a number of acoustical advantages are also given. A CIC is produced for the individual patient based on a silicon mold of the ear canal.

It is obvious that the space available inside a CIC hearing aid is severely limited. Hence, both the design of the internal components of the CIC and the placement and orientation of these are very critical as to whether it is actually possible to build a CIC for a given ear. Today the aforementioned designs are based on the experience and skills of the mechanical engineers in the hearing aid industry and a general knowledge about the anatomy and geometry of the ear. It is acknowledged that systematic knowledge of the geometry of ear canals and the variation thereof potentially could be extremely helpful in the mechanical design of new components for hearing aids.

To our knowledge no systematic description of the variation of the human ear canal across a population exists. Measurements of the anatomy of a single ear canal have been made for the purpose of prediction of sound-pressure

T. Dohi and R. Kikinis (Eds.): MICCAI 2002, LNCS 2489, pp. 373-380 2002.

(C) Springer-Verlag Berlin Heidelberg 2002 
level distribution [1]. Manufacturers of hearing aids have made initial testing of rapid prototyping of hearing aid shells using laser scans of ear impressions but have not performed statistical analysis of these. It is obvious that the systematic description of the variation of the shape of the ear canal must be done using statistical methods. In recent years shape analysis has been used in the description, identification and segmentation of biological shapes.

A popular method for building shape models is the Active Shape Model method by Cootes et al. 22. It is dependent of corresponding landmarks placed on the shapes in the training set. Most previous approaches to automated and semiautomated landmark generation and registration of 3D-surfaces use the local surface geometry. Examples of this are the non-rigid registration technique by Feldmar and Ayache [3], the local geometry and surface geodesic approach by Wang et al. 4] and the surface signature technique by Yamany et al. [5]. A method for automatic landmark generation based on a symmetric version of the iterative closest point algorithm is presented in [6. However, this method is dependent on the global variation of the shape and apparently does not handle boundary areas or areas that are not well defined for all shapes.

In this project the ear canals are represented as 3D-surfaces constructed by a laser scanner. The local surface geometry of the ear canals varies much from one individual to another and therefore only very few ridges and extremal points are stable when comparing groups of ear canals. The surfaces of the ear canals are not closed due to the opening of the ear canal and because the ear impressions are terminated in front of the eardrum. Automatic landmark generation and correspondence is therefore difficult to establish with methods based on surface geometry.

We have chosen to base our method on the assumption that it is possible to place anatomical landmarks on the ear canal. The anatomical landmarks do not constitute an exhaustive description of the surface of the ear canal and it is therefore necessary to generate a more dense set of landmarks describing the shape. Interpolating landmarks is straightforward in 2D, but in 3D no spatial ordering of landmarks is usually defined and therefore the interpolation is much more difficult.

A landmark based approach is found in [7] where a template mesh is deformed to each shape in the training set using a thin plate spline transformation based on the annotated landmarks. This is followed by a regularisation step where each vertex of the template mesh is adapted to the current shape without causing folds. This method is well suited for closed surfaces since it does not incorporate the possibility that patches of the surface are not well defined on all shapes in the training set.

A similar approach is found in $[8]$ with the addition that the method is able to handle surfaces with ill-defined areas. This is done by pruning the template mesh to only include the vertices that are well defined for all training shapes. In this method, the training shapes are warped to a template shape, and the correspondence is then made by projecting the vertices from the template shape to the warped training shapes. This is followed by an inverse warping of 
the adapted template shape, which gives the dense correspondence between the training shapes.

Our approach is similar to [8] but instead of warping each shape in the training set to the template mesh, the template mesh is warped to each shape in the training set eliminating the need for an inverse warp.

Other methods that could be well suited for the tubular-like ear canals are the spherical harmonics approach by Gerig [9] or the M-Rep approach by Pizer [10]. However, it is not clear how these methods will work on non-closed surfaces. A similar method that supports non-closed surfaces is the Fourier surfaces explored by Staib et al. 11 .

\section{Method}

The data were collected by laser scanning of a set of 29 ear impressions taken from 20 male and 9 female subjects. The surfaces are reconstructed using the Power Crust [12]. Each reconstructed surface contains approximately 20,000 vertices and 35,000 triangles. An example of a scanned ear impression is seen in Fig. 1. An anatomical description of the external ear and a formal naming convention used for ear impressions can be found in 1314 .

\subsection{Annotation of Anatomical Landmarks}

Using a custom-made surface annotation tool the third author, who is an expert of the anatomy of the ear canal has annotated the 29 ear canals. 18 anatomical landmarks are placed on each ear canal. The landmarks constitute a sparse correspondence between the surfaces of the ear canals in the training set.

The surface of the ear canal is not closed and therefore it is necessary to identify the invalid areas. Planes that separate the valid parts of the surface from the invalid parts are defined for that purpose. In Fig. 1] an ear canal with the anatomical landmarks and separating planes is seen.

\subsection{Surface Correspondence Using Thin Plate Spline Warping}

The anatomical landmarks do not constitute an exhaustive description of the surface of the ear canal and it is therefore necessary to generate a more dense set of landmarks describing the shape. For that purpose a template mesh is constructed and applied to all shapes in the training set.

The mesh constructed by the surface reconstruction contains far more vertices than needed to give a satisfactory shape description. To construct a template mesh that can be used as a basis for the further shape analysis a well defined ear canal surface is chosen and decimated by a standard algorithm 15 .

The anatomical landmarks and the template mesh are used to establish a dense surface correspondence between the shapes in the training set. Since the template mesh is made from one of the actual ear canals in the training set anatomical landmarks of this mesh exist. 
The template mesh is applied to each of the shapes in the training set using a Thin Plate Spline (TPS) warp based on the corresponding anatomical landmarks. TPS is a warp function that minimises the bending energy [16].

Since the TPS transform is only exact for the anatomical landmark locations, the vertices of the template mesh will not lie on the surface of the target shape. Finally, moving each vertex in the warped template mesh to the closest point on the target surface completes the dense correspondence. This introduces the risk of so called inversions, where the vertices of the template mesh shift place and cause folds in the mesh. Techniques to avoid this exist [17 7] but it has not been necessary here.

When the template mesh is warped to another shape, it can happen that some points are placed outside the valid area on the target shape. When warped to each shape in the training set, the template mesh must only cover valid areas. This is accomplished by investigating whether any points from the template mesh are warped into the areas marked as invalid by the separation planes. The template mesh is then pruned to contain only the points that are warped to valid areas for all shapes in the training set. The template mesh contains 3,000 vertices after decimation and pruning.

When the dense correspondence has been established the connectivity of the pruned template mesh can be applied to the points of correspondence on each shape to yield a set of new meshes. It is now possible to dispose of the anatomical landmarks as well as the original meshes of the training set. The set of meshes with dense correspondence is used in the following statistical shape analysis.

\subsection{Building the Point Distribution Model}

The set of corresponding meshes are aligned by a generalised Procrustes analysis [18. The pure shape model is built by using a similarity transformation in the Procrustes alignment while a rigid-body transformation is used to build the size-and-shape model [19].

Following the approach found in the Active Shape Model [2] a principal component analysis (PCA) is performed on the Procrustes aligned shapes. Each shape is represented as a vector of concatenated $\mathrm{x}, \mathrm{y}$ and $\mathrm{z}$ coordinates $\mathbf{x}_{i}=$ $\left[x_{i 1}, y_{i 1}, z_{i 1}, \ldots, x_{i n}, y_{i n}, z_{i n}\right]^{T}, i=1, \ldots, s$, where $n$ is the number of vertices and $s$ is the number of shapes. The PCA is performed on the shape matrix $\mathbf{D}=\left[\left(\mathbf{x}_{1}-\overline{\mathbf{x}}\right)|\ldots|\left(\mathbf{x}_{s}-\overline{\mathbf{x}}\right)\right]$, where $\overline{\mathbf{x}}$ is the average shape. The eigenvectors can be regarded as translation vectors that when added to the mean shape will deform the shape according to the modes of variation found in the training shapes. A new shape exhibiting the variance seen in the training set is made by adding a combination of eigenvectors to the average shape $\mathbf{x}_{n e w}=\overline{\mathbf{x}}+\Phi \mathbf{b}$, where $\mathbf{b}$ is a vector of weights controlling the modes of shape variation and $\Phi=\left[\phi_{1}\left|\phi_{2}\right| \ldots \mid \phi_{t}\right]$ is the matrix of the first $t$ eigenvectors. An arbitrary shape $\mathbf{x}^{\prime}$ aligned to the Procrustes average can be approximated by the shape model by projecting the residuals from the average shape onto the eigenvectors $\mathbf{b}=\Phi^{T}\left(\mathbf{x}^{\prime}-\overline{\mathbf{x}}\right)$. The resulting parameter vector $\mathbf{b}$ is used in the statistical analysis below. 


\section{Results}

\subsection{General Observations}

The three first modes of variation of the pure shape model are seen in Fig. 2 and the average shape is seen in Fig. 11 All the generated shapes look like real ear canals with no deformations or folds in the mesh. It is seen that the mode 1 deformation consists of a bending of the canal and a flattening of the concha part. Mode 2 explains some of shape variation seen in the inner part of the ear canal. Mode 3 is a combination of a flattening and twisting of the inner part of the ear canal and a general shape change of the concha.

The distribution of the modes against each other has been examined using pairwise plots, and no obvious abnormalities were found.
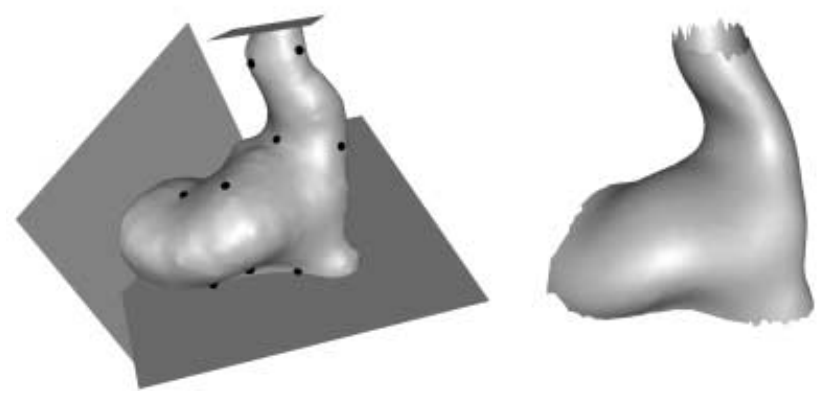

Fig. 1. To the left an example of a surface representation of an ear canal with the anatomical landmarks and the planes that separate the valid areas from the invalid areas. The thin structure in the top is the actual canal. The larger lower part is the concha. Only part of the concha is used and therefore a plane through concha is defined. To the right is the average shape from the pure shape model

\subsection{Classification of Surfaces}

Testing the validity and usability of the shape model is done by examining its ability to reflect gender related differences in the size and shape of the ear canals.

It is first examined if there is a systematic gender-related difference in the centroid sizes of the ear canals. This test is performed using the centroid sizes of the dense surface meshes of the training set calculated prior to the Procrustes alignment. The centroid size is the square root of the sum of squared Euclidean distances from each landmark to the center of mass (the centroid) [19. A standard t-test shows a highly significant difference in size between males and females $(\mathrm{p}=0,0003)$. The gender related difference in size corresponds to $9 \%$ of the average centroid size.

Testing for a shape difference between genders is done using the vertices from the Procrustes aligned dense surface meshes from the pure shape model, meaning that they are scaled to unit size. In this context, shape is thus unrelated 


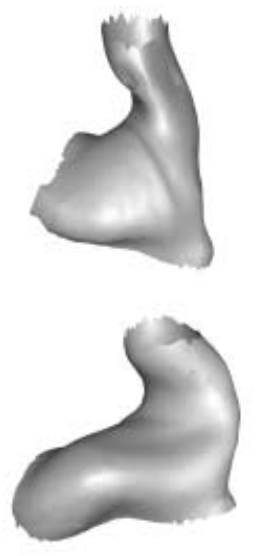

(a) Mode 1

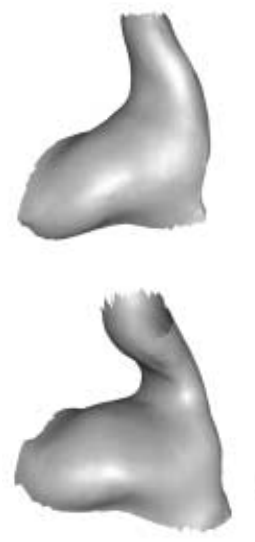

(b) Mode 2
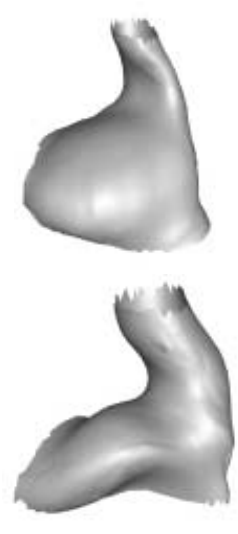

(c) Mode 3

Fig. 2. Pure shape model. Each shape has been generated by varying the first three modes of variation between -3 (top) and +3 (bottom) standard deviations

to size. In order to avoid the problem of multiple testing (as would be the case for stepwise selection, for example) the following procedure is adopted. First the dimensionality is reduced by a principal component analysis as described earlier, secondly the number of components to retain is chosen, and finally a multivariate analysis of variance [20] is performed on these components. A typical method for determining the number of principal components to retain is to include just enough components to explain some arbitrary amount (typically $98 \%$ ) of the variance. This criteria often results in far to many components being included in the further analysis and therefore Horn's parallel analysis [21] is chosen as a more objective way of deciding on how many components to include. The eigenvalues of the shapes are compared to those obtained for equivalent uncorrelated data, obtained by randomly scrambling each row in the shape matrix $\mathbf{D}$. In this way, the number of modes to retain is 7 as seen on the scree-plot in Fig. 3. A multivariate analysis of variance (which in this case is equivalent to Hotelling's $\mathrm{T}^{2}$ ) of these 7 principal component scores per shape although not strictly significant $(p=0,083)$ does indicate a shape difference between genders. Univariate analyses on the single principal components show that this is mainly due to the first mode of variation $(\mathrm{p}=0,0052)$. This is also seen in Fig. [3] where centroid size is plotted against mode 1 from the pure shape model. The exaggerated male-female mode shape variation is seen as the shape variation of mode 1 in Fig. 2 .

\section{Summary and Conclusions}

In this paper a method to generate dense surface distribution models of the human ear canal has been described. The generated models show consistency, 


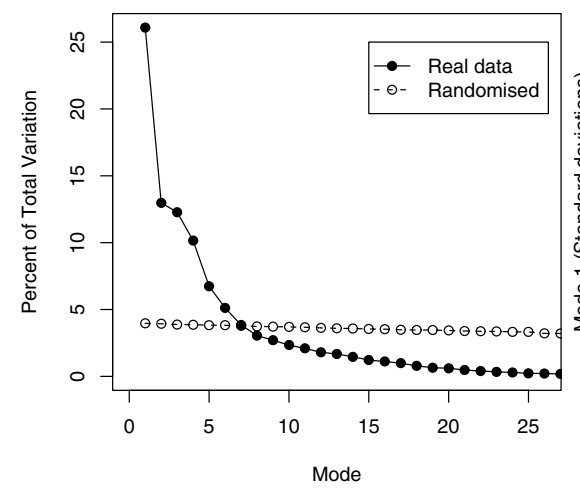

(a) Scree plot

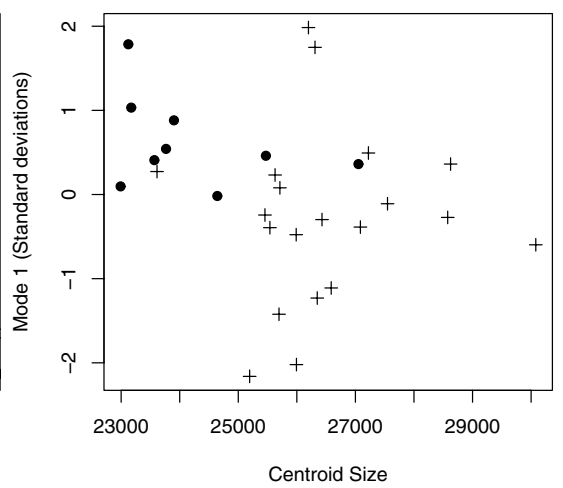

(b) Size and shape plot

Fig. 3. To the left is a plot of the eigenvalues of the shapes from the pure shape model, compared to those for a randomised version of the data (each row of the shape matrix $\mathbf{D}$ was scrambled). The lines are crossing approximately where mode $=7$. To the right is a plot of centroid size versus mode 1 from the pure shape model. The full dots are females while the plus signs are male. It is seen that both size and mode 1 separates males from females

and a large part of the variation found in the training data is explained by a small number of modes of variation. The method is general, and can be applied to all types of surfaces as long as it is possible to mark the valid areas of the surfaces and to place landmarks.

From an anatomical point of view it is interesting that the model is able to differentiate ear canals from males from ear canal from females based on both their size and their shape. The anatomical results based on this small data set are only strong enough to support general conclusions. For more detailed results a larger and more balanced data set is required.

The most important results of this paper are the proof-of-concept regarding the ability to build a meaningful statistical shape model of the human ear canal and that it is possible to describe a complex gender-related shape variation using a single parameter.

\section{References}

1. Stinson, M.R., Lawton, B.W.: Specification of the geometry of the human ear canal for the prediction of sound-pressure level distribution. J. Acoust. Soc. Am. 85 (1989) 2492-2503

2. Cootes, T., Cooper, D., Taylor, C., Graham, J.: Active shape models - their training and application. Computer Vision and Image Understanding 61 (1995) 38-59

3. Feldmar, J., Ayache, N.: Rigid, affine and locally affine registration of free-form surfaces. International Journal of Computer Vision 18 (1996) 99-119

4. Wang, Y., Peterson, B.S., Staib, L.H.: Shape-based 3D surface correspondance using geodesics and local geometry. Computer Vision and Pattern Recognition 2 (2000) 644-651 
5. Yamany, S.M., Farag, A.A.: Free-form surface registration using surface signatures. In: ICCV (2). (1999) 1098-1104

6. Brett, A., Taylor, C.: A method of automated landmark generation for automated 3D pdm construction. In: British Machine Vision Conference. (1998) 914-923

7. Lorenz, C., Krahnstöver, N.: Generation of point-based 3D statistical shape models for anatomical objects. Computer Vision and Image Understanding 77 (2000) 175191

8. Hutton, T., Buxton, B., Hammond, P.: Dense surface point distribution models of the human face. In: Proceedings of the Workshop on Mathematical Methods in Biomedical Image Analysis, CVPR (2001)

9. Gerig, G., Styner, M., Jones, D., Weinberger, D., Lieberman, J.: Shape analysis of brain ventricles using spharm. In: Workshop on Mathematical Methods in Biomedical Image Analysis, IEEE Computer Society (2001) 171-178

10. Pizer, S., Thall, A., Chen, D.: M-reps: A new object representation for graphics. Technical report, University of North Carolina at Chapel Hill (1999)

11. Staib, L.H., Duncan, J.S.: Model-based deformable surface finding for medical images. IEEE Trans. Medical Imaging 15 (1996) 720-731

12. Amenta, N., Choi, S., Kolluri, R.: The power crust. In: Proceedings of 6th ACM Symposium on Solid Modeling. (2001) 249-260

13. Alvord, L.S., Morgan, R., Cartwright, K.: Anatomy of an earmold: A formal terminology. J. Am. Acad. Audiol. 8 (1997) 100-103

14. Alvord, L.S., Farmer, B.L.: Anatomy and orientation of the human external ear. J. Am. Acad. Audiol. 8 (1997) 383-390

15. Schroeder, W., Zarge, J., Lorensen, W.: Decimation of triangle meshes. Computer Graphics 26 (1992) 65-70

16. Bookstein, F.: Shape and the information in medical images: A decade of the morphometric synthesis. Computer Vision and Image Understanding 66 (1997) $97-118$

17. Andresen, P., Nielsen, M.: Non-rigid registration by geometry constrained diffusion. In: Proceedings of Medical Image Computing and Computer Assisted Intervention. Volume 1679. (1999) 533-543

18. Gower, J.: Generalized Procrustes analysis. Psychometrika 40 (1975) 33-51

19. Dryden, I., Mardia, K.: Statistical Shape Analysis. Wiley, Chichester (1997)

20. Johnson, R.A., Wichern, D.W.: Applied Multivariate Statistical Analysis. PrenticeHall (1982)

21. Horn, J.L.: A rationale and test for the number of factors in factor analysis. Psychometrika 30 (1965) 179-186 